\title{
Comportamento Reológico de Xantana Produzida por Xanthomonas arboricola pv pruni para Aplicação em Fluido de Perfuração de Poços de Petróleo
}

\author{
Caroline D. Borges, Claire T. Vendruscolo \\ Centro de Biotecnologia, UFPel \\ André L. Martins, Rosana F. T. Lomba \\ Centro de Pesquisas e Desenvolvimento Leopoldo Américo Miguez de Mello, Petrobras S.A.
}

Resumo: O trabalho objetivou avaliar xantanas produzidas por Xanthomonas arboricola pv pruni como viscosificante de fluido para aplicação na perfuração de poços de petróleo, comparando-as com três amostras comerciais utilizadas para este fim. Para isto, a viscosidade de soluções aquosas e salinas $\left(\mathrm{NaCl}, \mathrm{KCl}\right.$ e $\left.\mathrm{CaCl}_{2}\right)$ das diferentes xantanas, em distintas temperaturas $\left(25,45,65\right.$ e $\left.80^{\circ} \mathrm{C}\right)$, foi determinada em reômetro. Os parâmetros reológicos índice de consistência (K) e o índice de fluxo (n) foram obtidos pelo ajuste do modelo de Ostwald-de-Waelle. A força gel $\left(\mathrm{G}_{0}, \mathrm{G}_{10}\right.$ e $\left.\mathrm{G}_{30}\right)$ foi determinada em viscosímetro, e a concentração de sais monovalentes (sódio e potássio) e divalentes (cálcio e magnésio) foi determinada em fotômetro de chama e em espectrômetro de absorção atômica, respectivamente. De uma forma geral, os resultados obtidos neste estudo mostram que as xantanas produzidas pelo patovar pruni, Xp 106-600 e Xp 115-600, por conterem baixa concentração de sais, apresentaram potencial para aplicação em fluido de perfuração de poços de petróleo, quando utilizada solução salina. Já os polímeros comerciais apresentaram melhores resultados quando utilizados em solução aquosa, pois continham alta concentração de sais.

Palavras-chave: Xanthomonas arboricola pv pruni, xantana, fluido de perfuração de poços de petróleo.

\section{Rheological Behaviour of Xanthan produced by Xanthomonas arboricola pv pruni for Application in Fluid of Oil Well Perforation}

\begin{abstract}
The aim of this work was to evaluate xanthans produced by Xanthomonas arboricola pv pruni to be used for increasing the viscosity of fluids applied in the perforation of oil wells, which were compared with three commercial samples. In order to do that, the viscosity of aqueous and saline solutions $\left(\mathrm{NaCl}, \mathrm{KCl}\right.$ and $\left.\mathrm{CaCl}_{2}\right)$ from different xanthans, at different temperatures $\left(25,45,65\right.$ and $\left.80{ }^{\circ} \mathrm{C}\right)$, was determined in a rheometer. The rheological parameters consistency index $(\mathrm{K})$ and flow behaviour index (n) were obtained by adjusting the data with the Ostwald-de-Waelle model. $\mathrm{Gel}_{\text {strength }}\left(\mathrm{G}_{0}\right.$, $\mathrm{G}_{10}$ e $\mathrm{G}_{30}$ ) was determined in a viscometer, and the concentration of monovalent (sodium and potassium) and divalent salts (calcium and magnesium) was determined in flame photometer and in atomic absorption spectrometer, respectively. Taken together, the results obtained in this study showed that xanthans produced by patovar pruni, Xp 106-600 and Xp 115-600, are promising for fluids used in oil well perforation with saline solutions, because they have low concentration of salts. As for the commercial polymers, they presented the best results when used in aqueous solution, because they had high salt concentrations.
\end{abstract}

Keywords: Xanthomonas arboricola pv pruni, xanthan, fluid for oil well perforation.

\section{Introdução}

Goma xantana é um polissacarídeo produzido por espécies de bactérias do gênero Xanthomonas, normalmente Xanthomonas campestris pv campestris ${ }^{[1]}$. Sua utilização como espessante e estabilizante tornou-se crescente com o passar dos anos, principalmente no campo petrolífero, onde é utilizada no fluido de perfuração e na recuperação terciária de poços de petróleo ${ }^{[2-4]}$.
Os fluidos de perfuração são misturas complexas de sólidos, líquidos e, por vezes, até gases. São tradicionalmente classificados, de acordo com seu constituinte principal, em fluidos à base de gás, à base de óleo e à base de água. A utilização de um ou outro tipo de fluido depende da profundidade do poço e do tipo de formação a ser perfurada ${ }^{[5]}$. Os fluidos à base de água são os mais utilizados na maioria das perfurações em todo mundo, por serem considerados

Autor para correspondência: Caroline D. Borges, Centro de Biotecnologia, Universidade Federal de Pelotas, Campus Universitário s/n, Caixa Postal 354, CEP: 96010-900, Pelotas, RS, Brasil. E-mail: caroldellin@bol.com.br 
ecologicamente seguros, biodegradáveis, apresentarem baixa toxicidade e baixa bioacumulação ${ }^{[6]}$. A principal função da água neste tipo de fluido é prover o meio de dispersão para os materiais coloidais, principalmente argilas e polímeros ${ }^{[5]}$. Para isto, os polímeros devem apresentar alta viscosidade em baixas concentrações, comportamento pseudoplástico e estabilidade da viscosidade à salinidade, temperatura e condições alcalinas ${ }^{[7]}$; de modo que apresente fácil injeção, mantenha os cascalhos em suspensão, facilitando a remoção destes e estabilize as paredes do poço ${ }^{[5]}$.

Os polímeros mais utilizados na formulação do fluido de perfuração à base de água eram a carboximetilcelulose - CMC, celulose polianiônica - PAC e poliacrilamida parcialmente hidrolisada - PHPA. Entretanto, estes polímeros são muito sensíveis às condições de alta salinidade, baixando sua eficiência ${ }^{[3,7]}$. Desta forma, a goma xantana passou a ser amplamente utilizada para este propósito, pois suas soluções são estáveis à variação do $\mathrm{pH}$, força iônica e temperatura ${ }^{[8,9]}$.

Apesar do grande mercado consumidor de xantana e de suas diferentes aplicações e potencialidades, poucos são os países que a produzem. A xantana utilizada no Brasil ainda é importada na sua totalidade, entretanto o país possui os insumos básicos utilizados durante o processo, como o açúcar de cana e o etanol, o que o torna um centro potencial para a produção deste polímero.

Este trabalho objetivou avaliar xantanas produzidas por Xanthomonas arboricola pv pruni como viscosificante de fluido para aplicação na perfuração de poços de petróleo, comparando-as com amostras comerciais.

\section{Experimental}

\section{Material}

Foram avaliadas xantanas purificadas produzidas por Xanthomonas arboricola pv pruni denominadas Xp 106-600, Xp 115-600 e Xp 115-400 e três xantanas, cedidas pela Petrobras, de utilização em fluido de perfuração de poços de petróleo, aqui nomeadas por amostras XC-A; XC-B e $\mathrm{XC}-\mathrm{C}$.

\section{Preparo dos fluidos}

Foram utilizados dois fluidos bases: A) solução aquosa de xantana (1,5 lb/bbl - libra/barril ou 4,3 g.L-1 $)$; B) solução salina de $\mathrm{NaCl}\left(21 \mathrm{lb} / \mathrm{bbl}\right.$ ou 60 g.L $\left.\mathrm{L}^{-1}\right), \mathrm{CaCl}_{2}(0,07 \mathrm{lb} / \mathrm{bbl}$ ou $\left.0,2 \mathrm{~g} . \mathrm{L}^{-1}\right), \mathrm{MgCl}_{2}\left(0,03 \mathrm{lb} / \mathrm{bbl}\right.$ ou $\left.0,08 \mathrm{~g} . \mathrm{L}^{-1}\right)$ adicionada de xantana $\left(1,5 \mathrm{lb} / \mathrm{bbl}\right.$ ou $\left.4,3 \mathrm{~g} . \mathrm{L}^{-1}\right)$. Os fluidos foram preparados em água deionizada, sendo o polímero adicionado à solução ao longo de 5 minutos, utilizando um misturador Hamilton Beach a 16.000 rpm. As soluções permaneceram sob agitação por mais 10 minutos e, após este período, foram acondicionadas em células de aço inox. As células foram armazenadas em estufa rotativa a $50{ }^{\circ} \mathrm{C}$, durante 16 horas. Após este período as amostras foram resfriadas a temperatura ambiente ${ }^{[10]}$.

\section{Métodos}

As curvas de viscosidade foram determinadas em reômetro (HAAKE modelo RS 600), no módulo rotativo, a 25, 45,65 e $80{ }^{\circ} \mathrm{C}$. Foram utilizados sistema de cilindro coaxial com sensor DG41 e taxa de deformação de 1 a $1000 \mathrm{~s}^{-1}$. Os parâmetros reológicos, índice de consistência (K) e o índice de fluxo (n) foram obtidos pelo ajuste do modelo de Ostwald-de-Waelle.

A força gel $\left(\mathrm{G}_{0}, \mathrm{G}_{10}\right.$ e $\left.\mathrm{G}_{30}\right)$ foi determinada em viscosímetro (FANN modelo 35A), conforme norma N-2605 da Petrobras ${ }^{[10]}$.

Os sais monovalentes (sódio e potássio) e divalentes (cálcio e magnésio) foram determinados em fotômetro de chama (Cole-Parmer modelo 2655-00) e espectrômetro de absorção atômica (Hitachi modelo Z8230), respectivamente. Para a realização destas análises, as amostras $(0,4 \mathrm{~g})$ foram calcinadas a $550{ }^{\circ} \mathrm{C}$ e as cinzas resultantes foram submetidas à digestão com $12 \mathrm{~mL}$ de água régia. Os sais formados foram diluídos a $100 \mathrm{~mL}$ pela adição de solução de $\mathrm{HCl} 1 \%{ }^{[11]}$.

\section{Resultados e Discussão}

A viscosidade de soluções aquosa e salina de diferentes xantanas foi avaliada em distintas temperaturas. As curvas de viscosidade obtidas são mostradas nas Figuras 1a, b, 4a, b, e referem-se ao modelo não ajustado. Após, através do ajuste do modelo de Ostwald-de-Waelle, foram determinados o índice de consistência (K) e o índice de fluxo (n). Este modelo foi aplicado por ser um dos mais utilizados para descrever o comportamento de fluidos em campos petrolíferos. O coeficiente de correlação, para todas as soluções, foi superior a 0,99 , independente da temperatura.

A viscosidade a $25^{\circ} \mathrm{C}$ das soluções aquosas das xantanas comerciais avaliadas foi superior àquelas produzidas pelo patovar pruni; na taxa de deformação de $60 \mathrm{~s}^{-1}$ foram obtidos os seguintes valores: $89,5 \mathrm{mPa} . \mathrm{s}$ para $\mathrm{XC}-\mathrm{A} ; 83,8 \mathrm{mPa}$.s para XC-B; 89,9 mPa.s para XC-C; 72,5 mPa.s para Xp 106-600; 73,2 mPa.s para Xp 115-600 e 68,1 mPa.s para Xp 115-400 (Figura 1a). Semelhantemente, mas com valores inferiores, nas temperaturas de 45 e $65^{\circ} \mathrm{C}$ as xantanas comerciais apresentaram viscosidades superiores àquelas produzidas pelo patovar pruni (resultados não mostrados). Na temperatura de $80{ }^{\circ} \mathrm{C}$ ocorreu uma redução acentuada de viscosidade para as soluções de xantanas produzidas pelo patovar pruni, alcançando na taxa de deformação de $60 \mathrm{~s}^{-1} 42,9 \mathrm{mPa}$.s para Xp 106-600; 38,3 mPa.s para Xp 115-600 e 42,9 mPa.s para Xp 115-400. As amostras comerciais, com exceção da XC-B, apresentaram viscosidade superior, em toda faixa de taxa de deformação avaliada, alcançando na taxa de deformação de $60 \mathrm{~s}^{-1}$ os valores de 71,5 mPa.s para XC-A, 74,2 mPa.s para XC-C e 36,2 mPa.s para XC-B (Figura 1b). Este polímero apresentou viscosidade inferior aos polímeros produzidos pelo patovar pruni a partir da taxa de deformação de $50 \mathrm{~s}^{-1}$.

Comportamento semelhante foi observado nos resultados de índice de consistência (Figura 2), que indica o grau de 


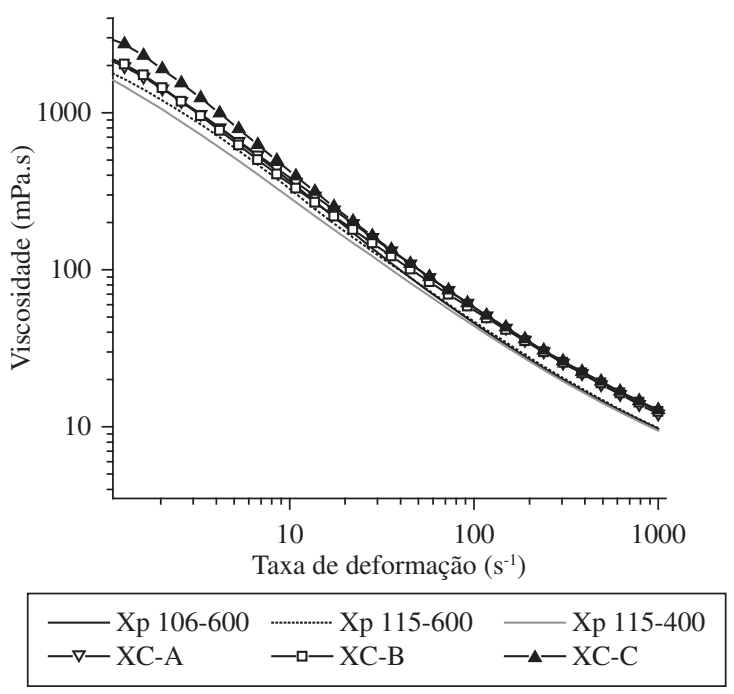

(a)

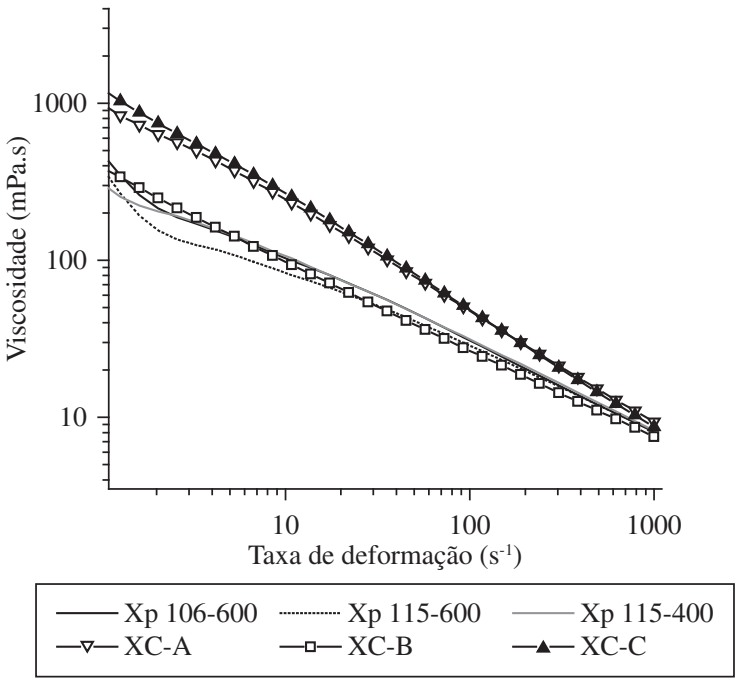

(b)

Figura 1. a) Curvas de viscosidade a $25^{\circ} \mathrm{C}$; e b) $80{ }^{\circ} \mathrm{C}$ de soluções aquosas de xantanas $(1,5 \mathrm{lb} / \mathrm{bbl})$.

resistência do fluido diante do escoamento, ou seja, quanto maior o valor de $\mathrm{K}$ mais viscoso será o fluido. Alto valor de $\mathrm{K}$ é desejável em fluidos de perfuração, pois alta viscosidade é necessária para manter em suspensão os cascalhos gerados durante a perfuração ${ }^{[7,12]}$.

A Figura 3 mostra os resultados do índice de fluxo das soluções aquosas das xantanas em distintas temperaturas. Todas as soluções apresentaram comportamento pseudoplástico, entretanto, o aumento da temperatura diminuiu a pseudoplasticidade destas soluções. Até $45^{\circ} \mathrm{C}$, as soluções dos polímeros comerciais apresentaram maior pseudoplasticidade; entretanto, em 65 e $80{ }^{\circ} \mathrm{C}$, as soluções das amostras XC-A e XC-B, assim como Xp 115-400, apresentaram queda de pseudoplasticidade. Nesta faixa de temperatura a solução do polímero XC-C, seguida da Xp 106-600 e Xp 115-600, foram as mais pseudoplásticas.

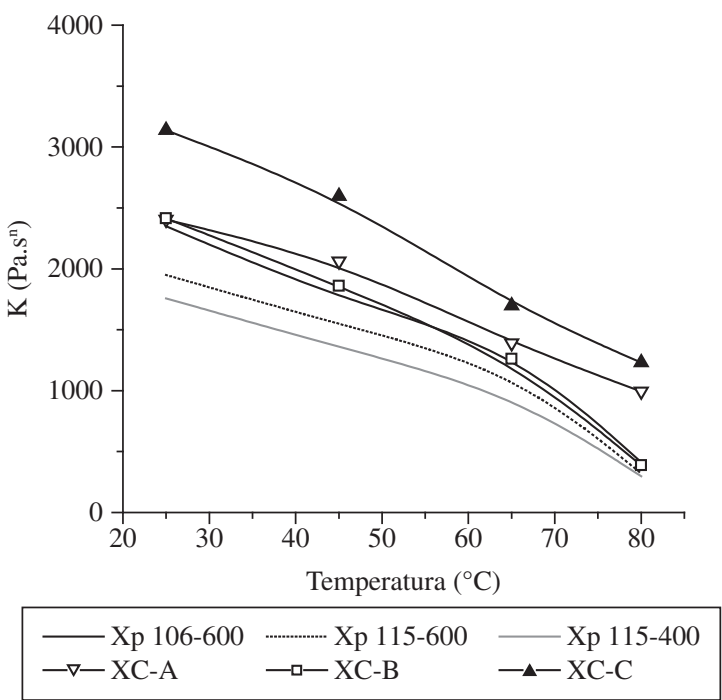

Figura 2. Índice de consistência em distintas temperaturas de soluções aquosas de xantanas $(1,5 \mathrm{lb} / \mathrm{bbl})$.

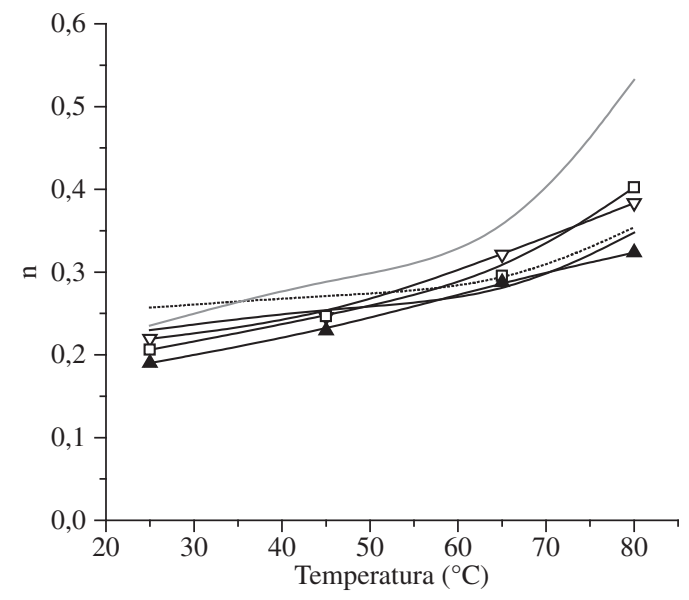

\begin{tabular}{|c|c|c|}
\hline $\begin{array}{l}-\mathrm{Xp} \mathrm{106-600} \\
-\nabla-\mathrm{XC}-\mathrm{A}\end{array}$ & $\begin{array}{l}\cdots-X p 115-600 \\
-\square-X C-B\end{array}$ & $\begin{array}{l}- \text { Xp } 115-400 \\
-\wedge-\text { XC-C }\end{array}$ \\
\hline
\end{tabular}

Figura 3. Índice de fluxo em distintas temperaturas de soluções aquosas de xantanas $(1,5 \mathrm{lb} / \mathrm{bbl})$.

O índice de fluxo (n) caracteriza o comportamento nãoNewtoniano e quando $\mathrm{n}<1$ o fluido é pseudoplásico. Baixos valores de $\mathrm{n}$ são desejáveis em fluidos de perfuração para facilitar a injeção do fluido e melhorar a taxa de perfuração. Cabe salientar que os parâmetros reológicos $\mathrm{K}$ e n são dependentes da concentração de xantana e das características do polímero ${ }^{[13]}$.

Em solução salina, diferentemente, as viscosidades a $25{ }^{\circ} \mathrm{C}$ das soluções de xantanas Xp 106-600 e Xp 115-600 foram superiores às demais, alcançando, respectivamente, 81,6 e 80,1 mPa.s na taxa de deformação de $60 \mathrm{~s}^{-1}$. A viscosidade da solução salina da amostra comercial XC-A foi similar a amostra Xp 115-400 até a taxa de deformação de $30 \mathrm{~s}^{-1}$, e ambas superiores as amostras comerciais XC-B (18,5 mPa.s) e XC-C (34,3 mPa.s) (Figura 4a). Comportamento semelhante, mas com valores inferiores em toda faixa de taxa de defor- 


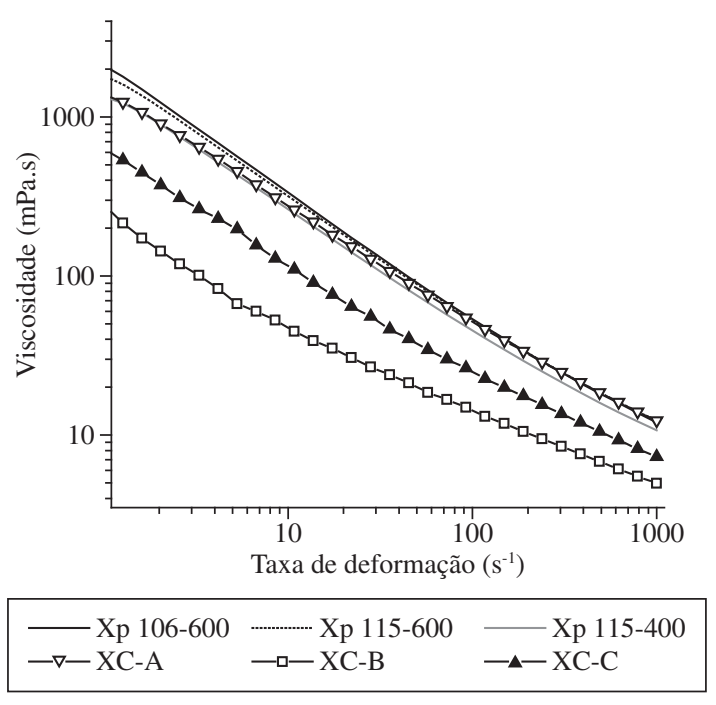

(a)

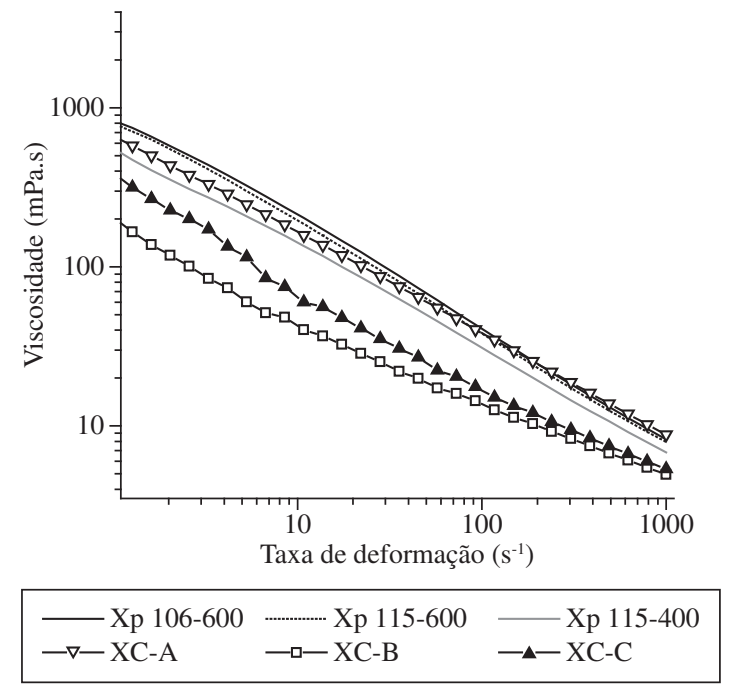

(b)

Figura 4. a) Curvas de viscosidade a $25{ }^{\circ} \mathrm{C}$; e b) $80{ }^{\circ} \mathrm{C}$ de soluções salinas de xantanas $(1,5 \mathrm{lb} / \mathrm{bbl})$.

mação, foi observado nas curvas de viscosidade a 45 e $65^{\circ} \mathrm{C}$ (resultados não mostrados) e $80^{\circ} \mathrm{C}$ (Figura $4 \mathrm{~b}$ ).

Os resultados do índice de consistência das soluções salinas das diferentes xantanas, mostrados na Figura 5, corroboram com aqueles obtidos nas Figuras $4 a$, $b$.

Em solução salina, apenas a solução da amostra XC-B manteve seu valor de pseudoplasticidade com o aumento da temperatura; nas demais soluções, a pseudoplastidade diminuiu (Figura 6). Até $45{ }^{\circ} \mathrm{C}$ as soluções das amostras Xp 106-600 e Xp 115-600 apresentaram maior pseudoplasticidade. Com o incremento da temperatura, a solução da XC-B mostrou-se mais pseudoplástica; entretanto, as xantanas do patovar pruni, Xp 106-600 e Xp 115-600, ainda apresentaram maior pseudoplasticidade que as demais comerciais.

Os resultados da força gel apresentados na Tabela 1 mostram que, em solução aquosa, todos os polímeros estão em conformidade com os valores especificados na norma N-2604

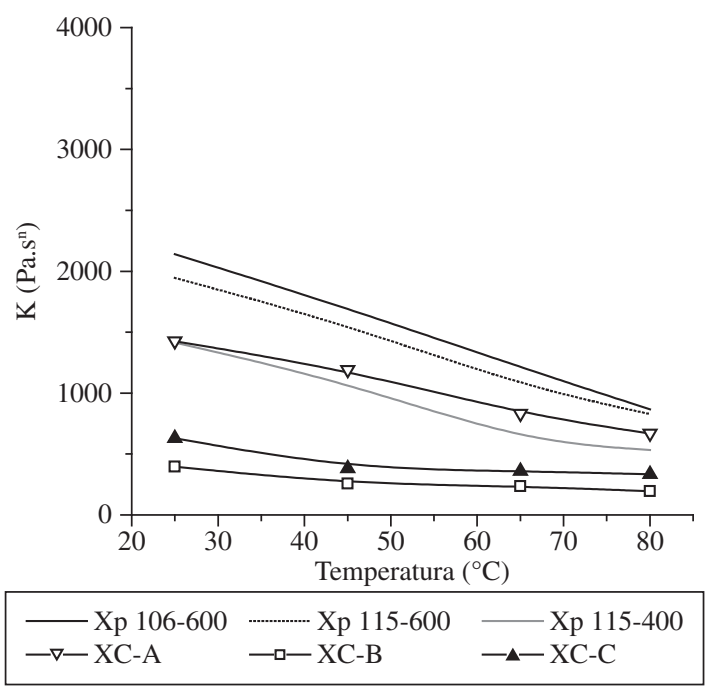

Figura 5. Índice de consistência em distintas temperaturas de soluções salinas de xantanas $(1,5 \mathrm{lb} / \mathrm{bbl})$.

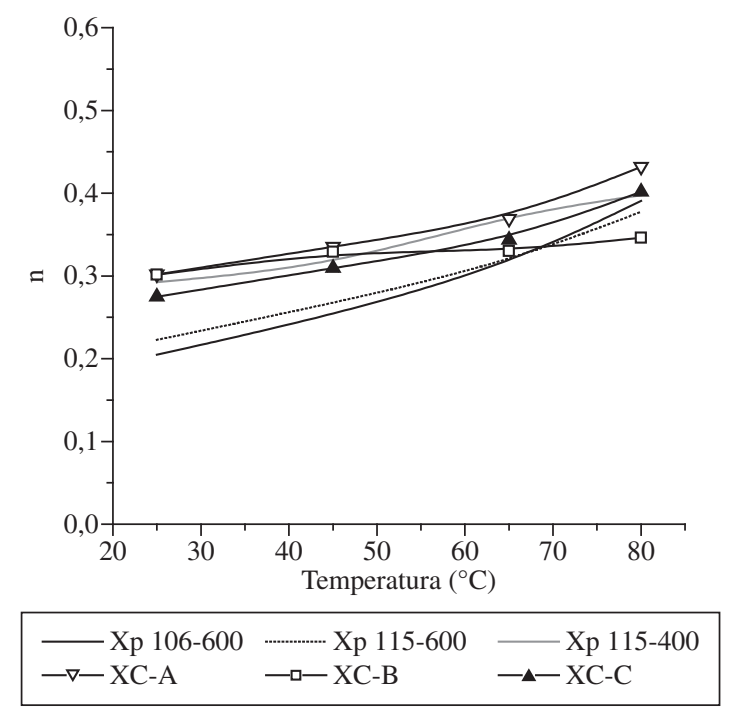

Figura 6. Índice de fluxo em distintas temperaturas de fluidos em solução salina de xantanas $(1,5 \mathrm{lb} / \mathrm{bbl})$.

Tabela 1. Resultados de força gel de fluidos em solução aquosa e salina adicionados de diferentes xantanas $(1,5 \mathrm{lb} / \mathrm{bbl})$.

\begin{tabular}{crcc}
\hline Amostra & \multicolumn{3}{c}{ Força gel (lbf/100 f t t $\mathbf{~}^{\mathbf{2}}$} \\
\cline { 2 - 4 } & $\mathbf{G}_{\mathbf{0}}$ & $\mathbf{G}_{\mathbf{1 0}}$ & $\mathbf{G}_{\mathbf{3 0}}$ \\
\hline Solução aquosa & & & \\
Xp 106-600 & 9 & 13 & 14 \\
Xp 115-600 & 9 & 13 & 14 \\
Xp 115-400 & 8 & 11 & 12 \\
XC-A & 10 & 13 & 13 \\
XC-B & 12 & 13 & 13 \\
XC-C & 12 & 15 & 16 \\
Solução salina & & & \\
Xp 106-600 & 9 & 12 & 13 \\
Xp 115-600 & 8 & 10 & 11 \\
Xp 115-400 & 7 & 10 & 10 \\
XC-A & 8 & 9 & 9 \\
XC-B & 3 & 5 & 5 \\
XC-C & 3 & 4 & 4 \\
\hline
\end{tabular}


da Petrobras ${ }^{[14]}$. Esta norma estabelece, em $\mathrm{lbf} / 100 \mathrm{ft}^{2}$, valor mínimo para $\mathrm{G}_{0}$ de $7 ; \mathrm{G}_{10}$ entre 10 e 15 e $\mathrm{G}_{30}$ máximo de 20 . Entretanto, em solução salina, apenas os polímeros produzidos pelo patovar pruni e a amostra comercial XC-A estão condizentes com estes valores.

A força gel também é um parâmetro de natureza reológica, que indica o grau de gelificação. A força gel $G_{0}$ mede a resistência inicial para colocar o fluido em fluxo. A força gel final, neste caso $\mathrm{G}_{30}$, mede a resistência do fluido para reiniciar o fluxo quando este fica um certo tempo em repouso devido a manobras e conexões. A diferença entre as forças géis indica o grau de tixotropia do fluido ${ }^{[5]}$. O comportamento tixotrópico é desejado para evitar a sedimentação dos sólidos perfurados. Por outro lado, se a viscosidade aumentar continuamente durante o tempo em que a perfuração pára, picos de pressão podem ser gerados quando a circulação recomeçar, e a pressão de fratura poderá ser alcançada. Desta forma, gelificação rápida e não progressiva é requerida ${ }^{[15]}$.

A água é a fase contínua e o principal componente de qualquer fluido à base de água, podendo ser doce, dura ou salgada. A água doce, por definição, apresenta salinidade inferior a $1000 \mathrm{ppm}$ de $\mathrm{NaCl}$ equivalente. Do ponto de vista industrial, para aplicação em fluido de perfuração, a água doce não necessita de pré-tratamento químico porque praticamente não afeta o desempenho dos aditivos empregados no preparo do fluido. A água dura tem como característica principal a presença de sais de cálcio e de magnésio dissolvidos, em concentrações suficientes para alterar o desempenho dos aditivos químicos. A água salgada é aquela com salinidade superior a $1000 \mathrm{ppm}$ de $\mathrm{NaCl}$ equivalente; pode ser natural, como a água do mar, ou pode ser salgada com a adição de $\mathrm{NaCl}, \mathrm{KCl}$ ou $\mathrm{CaCl}_{2}^{[5]}$.

De uma forma geral, os resultados obtidos neste estudo mostram que os polímeros comerciais apresentaram melhores resultados quando utilizados em solução aquosa. $\mathrm{O}$ mesmo comportamento foi observado por Correia, França e Mothé ${ }^{16]}$. Estes autores avaliaram o comportamento reológico de diferentes polímeros comerciais (xantana, poliacrilamida e guar) em água destilada e do mar, visando a sua utilização na indústria do petróleo. Em seu trabalho a goma xantana comercial utilizada também apresentou maior valor de viscosidade em água destilada, assim como a poliacrilamida. Já às soluções de goma guar foram mais viscosas em água do mar.

Entretanto, para a realidade brasileira, onde grande parte da exploração ocorre em alto mar, muitas vezes a água mari- nha é utilizada. Em determinadas situações pode-se utilizar a água adicionada de $\mathrm{NaCl}, \mathrm{KCl}$ e $\mathrm{CaCl}_{2}$. Nestas condições, as amostras Xp 106-600 e Xp 115-600 apresentaram maior capacidade viscosificante, em toda a faixa de temperatura avaliada, e também valores adequados para a força gel. A influência da temperatura alcançada durante a perfuração, na capacidade viscosificante do polímero, é outro parâmetro de grande importância. Comumente, a temperatura varia entre a ambiente, na superfície, até $60{ }^{\circ} \mathrm{C}$, no fundo do poço. Ainda existem poços cujas temperaturas são altas e podem alcançar valores acima de $150{ }^{\circ} \mathrm{C}^{[17]}$. Dessa forma, é interessante que o polímero utilizado mantenha sua alta capacidade viscosificante na faixa de temperatura em que será utilizado, mesmo na presença de sais. Apenas o polímero produzido pelo patovar pruni e a xantana comercial XC-A apresentaram esta característica.

O conteúdo de sais monovalentes, sódio e potássio, e divalentes, cálcio e magnésio, das diferentes xantanas foi determinado (Tabela 2). As amostras comerciais apresentaram um conteúdo de sais superior em relação às xantanas do patovar pruni.

A extensão da queda de viscosidade das soluções aquosas com o aumento da temperatura foi influenciada pela concentração de sais presente no polímero. As amostras comerciais XC-A e XC-C, em solução aquosa, apresentaram menor queda com o aumento da temperatura, provavelmente por apresentarem um conteúdo de sais divalentes superior às demais. Já a viscosidade da solução aquosa a $80{ }^{\circ} \mathrm{C}$ da amostra comercial XC-B e dos polímeros do patovar pruni foi inferior, decorrente do baixo conteúdo de sais divalentes.

Sabe-se que na presença de sais a temperatura de transição conformacional da xantana aumenta mantendo, dessa forma, a estrutura ordenada em temperaturas superiores e, com isso, mantém-se a viscosidade ${ }^{[18,19]}$. Entretanto, sais divalentes, como $\mathrm{CaCl}_{2}$ e $\mathrm{MgCl}_{2}$, são mais efetivos no aumento da temperatura de transição que sais monovalentes, como $\mathrm{NaCl}^{[3,18]}$. Segundo Holzwarth ${ }^{[18]}$ e Milas e Rinaudo ${ }^{[19]}$ em baixa concentração salina a viscosidade da solução de xantana diminui, pois a temperatura de transição conformacional é alcançada. Por essa razão, o polímero xantana comercializado para aplicação em fluido de perfuração contém produtos químicos para melhorar/estabilizar as propriedades reológicos em condições extremas ${ }^{[7]}$.

Em solução salina a viscosidade das amostras comerciais, que apresentam maior teor sais, foi inferior em relação à solução dos polímeros produzidos pelo patovar pruni.

Tabela 2. Conteúdo de sais mono e divalentes das diferentes xantanas analizadas.

\begin{tabular}{cccccccc}
\hline Xantana & $\mathbf{N a}(\%)$ & $\mathbf{K}(\%)$ & Cátions monovalentes $(\%)$ & $\mathbf{C a}(\%)$ & $\mathbf{M g}(\%)$ & Cátions divalentes (\%) & Cátions totais (\%) \\
\hline Xp 106-600 & 1,66 & 0,35 & 2,01 & 0,05 & 0,20 & 0,25 & 2,26 \\
Xp 115-600 & 1,68 & 0,35 & 2,03 & 0,05 & 0,24 & 0,29 & 2,32 \\
Xp 115-400 & 1,38 & 0,36 & 1,74 & 0,05 & 0,28 & 0,33 & 2,07 \\
XC-A & 0,47 & 0,42 & 0,89 & 1,55 & 0,06 & 1,61 & 2,50 \\
XC-B & 0,89 & 1,57 & 2,46 & 0,82 & 0,04 & 0,86 & 3,32 \\
XC-C & 0,27 & 1,44 & 1,71 & 1,50 & 0,07 & 1,57 & 3,28 \\
\hline
\end{tabular}


Pois, em altas concentrações salinas, independente da temperatura, ocorre uma mudança na conformação da molécula de xantana, de expandida para uma forma compactada. Esta mudança na dimensão hidrodinâmica da molécula causa um decréscimo da viscosidade da solução ${ }^{[18,20]}$. Comportamento semelhante foi observado por Xie e Lecourtier ${ }^{[3]}$. Estes autores avaliaram o comportamento reológico de uma suspensão de argila e xantana comercial na presença de $\mathrm{NaCl}$ ou $\mathrm{CaCl}_{2} \mathrm{e}$ sua estabilidade em diferentes temperaturas. De acordo com seus resultados, em baixa concentração de $\mathrm{NaCl}\left(1 \mathrm{~g} . \mathrm{L}^{-1}\right)$ não houve variação de viscosidade entre 30 e $70{ }^{\circ} \mathrm{C}$, entretanto, a $90^{\circ} \mathrm{C}$, a viscosidade foi inferior. Já em altas concentrações de $\mathrm{NaCl}\left(50\right.$ g. $\left.\mathrm{L}^{-1}\right)$, a viscosidade, embora inferior, também não variou com o aumento da temperatura entre 30 e $70{ }^{\circ} \mathrm{C}$. Apenas em $90{ }^{\circ} \mathrm{C}$ a viscosidade da suspensão em alta concentração salina foi superior àquela em baixa. Os autores também avaliaram a influência do $\mathrm{CaCl}_{2}$. Os resultados obtidos foram semelhantes, entretanto, em baixas concentrações, o decréscimo de viscosidade com o aumento da temperatura foi de menor relevância. Os autores concluíram que, para a utilização da suspensão em altas temperaturas, é necessária alta salinidade para evitar a degradação da molécula de xantana, ainda que a viscosidade seja reduzida nestas condições.

\section{Conclusão}

De uma forma geral, os resultados obtidos neste estudo mostram que as xantanas produzidas pelo patovar pruni, Xp 106-600 e Xp 115-600, contém baixa concentração de sais e apresentam comportamento reológico esperado para aplicação em fluido de perfuração de poços de petróleo quando utilizadas em solução salina, independente da temperatura. Já os polímeros comerciais cedidos pela Petrobras, apresentam melhores resultados apenas quando utilizados em solução aquosa, pois contém alta concentração de sais, não apresentando comportamento reológico desejado quando em soluções salinas.

\section{Agradecimentos}

O presente trabalho foi realizado com o apoio do Conselho Nacional de Desenvolvimento Científico e Tecnológico - CNPq - Brasil.

\section{Referências Bibliográficas}

1. Sutherland, I. W. - "Xanthan", in: Xanthomonas, Swings, J. G. \& Civerolo, E. L. (eds.), Chapman \& Hall, London (1993).

2. Kulicke, W. M.; Oertel, R.; Otto, M.; Kleinitz, W. \& Littmann, W. - Erdöl \& Kohle, Erdgas, Petrochemie, 43, p.471 (1990).
3. Xie, W. \& Lecountier, J. - Polym. Degrad. Stabil, 38, p.155 (1992).

4. Gallino, G.; Migliori, M. \& Cindio, B. - Rheol. Acta, 40, p.196 (2001).

5. Thomas, J. E. - "Fundamentos de Engenharia de Petróleo", Interciência, Rio de Janeiro (2001).

6. Amorim, L. V.; Farias, K. V.; Viana, J. D.; Barbosa, M. I. R.; Pereira, E.; França, K. B.; Lira, H. L. \& Ferreira, H. C. - Cerâmica, 51, p.128 (2005).

7. Shah, A. K. \& Ashtaputre, A. A. - J. Ind. Microbiol. Biotechnol., 23, p.442 (1999).

8. Rocks, J. K. - Food Technol., 25, p.476 (1971).

9. Born, K.; Langendorff, V. \& Boulenguer, P. - "Xanthan", in: Biopolymers, Steinbüchel, A.; Vandamme, E. J. \& De Baets, S. (eds.), Weley-VCH, Weinheim (2002).

10. Petrobras - "Ensaio de viscosificante para fluidos usados na exploração e produção de poços de petróleo e gás - Método de ensaio", N-2605 (2003).

11. American Society for Testing and Materials. "Annual Book of ASTM Standards ASTM D1428-64", West Conshohocken (1981).

12. Machado, J. C. V. - "Reologia e Escoamento de Fluidos Ênfase na Indústria do Petróleo", Interciência, Rio de Janeiro (2002).

13. Candia, J. L. F. \& Deckwer, W. D. - Biotechnol. Progr., 15, p.446 (1999).

14. Petrobras - "Viscosificante para fluidos usados na exploração e produção de poços de petróleo e gás - Especificação", N-2604 (2003).

15. Waldmann, A. T. A.; Branco, M. A. \& Martins, A. - "Rheological characterization of polymer solutions for oil well drilling applications", in: Proceedings of the XI International Macromolecular Colloquium, Gramado - RS, (2007).

16. Correia, D. Z.; França, F. P. \& Mothé, C. G. - Conjuntura \& Informação, 26, p.9 (2004).

17. Monteiro, V. A. R.; Martins, A. L.; Brandão, E. M. \& Mello, E. M. C. - "Estudo das propriedades reológicas de fluidos de perfuração de poços em águas profundas", in: Proceedings of the II Brazilian Conference on Rheology, Rio de Janeiro - RJ, (2004).

18. Holzwarth, G. - Biochemistry, 15, p.4333 (1976).

19. Milas, M. \& Rinaudo, M. - Carbohydrate Res., 158, p.191 (1986).

20. Carrigton, S.; Odell, J. \& Fisher, L. - Polymer, 37, p.2871 (1996).

Enviado: $21 / 11 / 08$

Reenviado: 07/03/09

Aceito: $17 / 03 / 09$ 